\title{
Associations Between Emotions: Correspondence Across Different Types of Data and Componential Basis
}

\author{
PETER KUPPENS,* IVEN VAN MECHELEN, DIRK J. M. SMITS \\ and PAUL DE BOECK \\ University of Leuven, Belgium
}

\begin{abstract}
Two issues regarding associations between negative emotions are addressed: (i) whether different types of data imply similar associations and (ii) whether these associations can be accounted for by the overlap between emotion components. In study 1, issue (i) was examined using individual differences covariation, situational covariation, and judged emotion similarity data. Results showed that the associations implied by the different data types were highly similar. Regarding issue (ii), we argue theoretically that component overlap may account for the obtained correspondence. Study 2 empirically demonstrated that the different types of association were strongly related to overlap in appraisals and action tendencies. Similarity judgments, however, seemed to be primarily based on emotion co-occurrence, and were only to a smaller degree directly related to component overlap. Copyright (C) 2004 John Wiley \& Sons, Ltd.
\end{abstract}

Over the past decades, a large amount of research has accumulated regarding structural representations of the interrelations between emotions. Across various studies, however, this research has been based upon different types of data on emotion associations, predominantly individual differences or situational emotion covariation data and cognitive-semantic data such as similarity ratings.

A first question that will be addressed in the present paper is whether or not these different types of data imply similar associations between emotions. As will be shown, from a theoretical point of view, this question is not trivial, because emotion associations can be expected to be both similar and dissimilar across different data types. In addition, up to now this question has not been fully straightened out empirically-findings are inconclusive; moreover, relations between some data types have not yet been investigated. In study 1 we made an attempt to clarify this situation by comparing different types of emotion association data: individual differences covariation, situational covariation, and similarity ratings.

*Correspondence to: Peter Kuppens, Department of Psychology, University of Leuven, Tiensestraat 102, 3000 Leuven, Belgium. E-mail: peter.kuppens@psy.kuleuven.ac.be

Contract/grant sponsor: Research Fund of the University of Leuven.

Contract/grant number: GOA/00/02. 
Findings on the similarity of the resulting emotion associations may be of utmost importance, because they may imply an important cue for tracing the role of the processes underlying emotions. Indeed, we will show on a theoretical level that, if an emotion is considered the resultant of a number of more elementary emotion components, then emotion associations implied by both individual differences and situational covariation data may be predicted to reflect component overlap between emotions. The latter then implies that both types of covariation data indeed may be expected to yield similar emotion associations. Furthermore, if similarity ratings are based on a comparison of emotion components, then similarity judgments should reflect component overlap as well, and such judgments should therefore imply similar emotion associations as both types of covariation data. On an empirical level, we will also address the issue of emotion components. Specifically, in study 2, it will be examined whether the various types of emotion association data from study 1 are related to component overlap of the emotions under study, making use of appraisals and action tendencies as emotion components.

In the present article, these issues are examined for negative emotions. We believe the relations we find will be more robust because they were found with respect to emotions of the same (negative) valence only: If one considered emotions of positive and negative valence simultaneously, then it would be expected that all measures (covariation, similarity, overlap) would be subject to the same valence-related source of variance, yielding an increase of all associations under study.

It should be noted that the studies reported in this paper partly overlap with respect to the data that were collected. In particular, the data on individual differences covariation, situational covariation, and similarity are the same across both studies. The studies are presented, though, as different studies in order to maintain the rationale behind the reported research.

\section{PART 1: CORRESPONDENCE BETWEEN EMOTION ASSOCIATIONS IMPLIED BY DIFFERENT TYPES OF DATA}

The data that are used in emotion structure research are of various kinds. Some of the existing research relies on covariances or correlations between emotions. Typically, researchers make use of covariation across persons of self-reported frequencies of experienced emotions (e.g., Diener, Smith, \& Fujita, 1995), or momentary affects (e.g., Russell, 1980; Watson \& Tellegen, 1985; Zevon \& Tellegen, 1982). Such covariations are based on individual differences in experienced emotions and will therefore be referred to as individual differences covariation data. We would like to note, however, that, in specific empirical studies, covariations of momentary experienced emotions across persons may be based not only on individual differences variance but also on situational variance, in particular if different persons are embedded in different contexts when providing their emotion ratings. In the latter case, the two sources of variance are confounded.

Alternatively, one could also examine the co-occurrence or covariation of emotions across different types of situations, which will henceforth be referred to as situational covariation data. This type of data has been much less used in emotion structure research. As argued by Lazarus (1994), however, it provides a distinct but equally valuable type of information as compared with individual differences covariation data, as it reflects variation across situational conditions in experienced emotions (unlike individual differences covariation data, which reflects variation across persons in the propensity to experience emotions). In addition, situational covariation data directly relates to the topic 
of mixed emotions or emotion blends, which has received a substantial amount of attention in recent emotion research (e.g. Coren \& Russell, 1992; Smith, 2002; Williams \& Aaker, 2002; Zelenski \& Larsen, 2000).

Finally, other studies have started from semantic emotion data-in particular similarity, prototypicality, or other ratings of emotional stimuli that aim to uncover the cognitive organization of people's knowledge about emotions (e.g., Morgan \& Heise, 1988; Russell, 1980; Shaver, Schwartz, Kirson, \& O’Connor, 1987). In this article, we focus on similarity data.

As mentioned above, different types of data are being used to study emotion associations. The question arises, however, whether these different types of data imply similar relations between emotions. The answer to this question is not straightforward, either theoretically or empirically. Theoretically, arguments in favour of similar as well as dissimilar associations across the different data types can be advanced. We will now do so for each combination of two types of data.

\section{Individual differences and situational covariation data}

There is no a priori reason to assume that the two types of covariation data would correspond. Depending on the nature of personality and situational influences on emotional experience, one could expect both similar and dissimilar emotion associations.

This can be demonstrated by means of two hypothetical examples. As a first example, let us assume that the main individual differences variable with respect to negative emotions is negative affectivity. Negative affectivity (along with positive affectivity) is considered to be an important determinant of mood and emotional experience (Watson, 2000) and can be hypothesized to be an important personality variable underlying the observed co-occurrence of emotions of the same negative hedonic valence across individuals (see, e.g., Diener, 1999). Let us further assume that the main situational influence on emotional experience is based on the situation's general emotion-eliciting power (comparable to, for example, motivational relevance; Smith \& Lazarus, 1993), which could be considered as a prerequisite for the occurrence of emotion blends. In this case, one may expect positive associations between negative emotions across persons as well as across situations, yielding similar emotion associations for the two data types. As a second example, let us again assume that negative affectivity is the main individual differences variable, but that different emotions are elicited by different situations - for instance because they are linked in specific ways to different motives (Zurbriggen \& Sturman, 2002). Specifically, assume for instance that achievement-related situation $X$ mainly elicits emotions $\mathrm{A}$ and $\mathrm{B}$ and that affiliation-related situation Y mainly elicits emotions $\mathrm{C}$ and $\mathrm{D}$ and so on. In this case, one may expect quite dissimilar emotion associations across persons compared with associations across situations. The general principle underlying these examples is the following: if the personality and situational effects on emotional experience are of a similar formal nature (as in the first example-a main effect of persons and main effect of situations on all emotions), then one can expect to find correspondence between the emotion associations as implied by the two data types, whereas when the two influences do not correspond (as in the second example-main effect of persons but situation $\times$ emotion interaction effect), one would not expect to obtain correspondence between the two sets of associations.

\section{Individual differences covariation and similarity data}

Correlations between self-ratings of emotions are generally accepted as a valid source of information on the individual differences covariation of emotions, whereas similarity data 
are assumed to stem from semantic knowledge (for a recent overview see, for example, Feldman Barrett \& Fossum, 2001). As such, it can be expected that the two types of data do not necessarily imply similar emotion associations. Alternatively, some authors have argued that individual differences covariation and similarity data contain essentially the same structural information, either because the processes underlying emotional experience are similar to those that structure emotion knowledge (Russell, 1980) or because similarity judgments reflect a person's intuitive impressions of emotion covariation (Reisenzein \& Schimmack, 1999; Schimmack \& Reisenzein, 1997).

\section{Situational covariation and similarity data}

Situational covariation data on emotions is assumed to reflect the situational co-occurrence of emotions in everyday life. Assuming again that similarity judgments reflect semantic emotion knowledge, it is not straightforward to expect that both types of data correspond with respect to their implied emotion associations. As mentioned above, however, Schimmack and Reisenzein (1997) have claimed that similarity judgments are essentially based on the degree of co-occurrence of emotions in everyday life (thus on episodic knowledge instead of on semantic knowledge), expecting a fair amount of correspondence between the two types of data.

Empirical findings on these issues are partial and rather inconclusive-partial, because the similarity between individual differences and situational covariation has not yet been investigated, and inconclusive, because previous research has yielded divergent findings. Regarding individual differences covariation and similarity data, structural models resulting from analyses based on both data types are sometimes quite different (for an overview, see Reisenzein \& Schimmack, 1999). Resenzein and Schimmack (1999), however, also compared raw data of both types and found a high degree of similarity between them. They further argued that differences between structural models are mainly due to methodological artifacts such as the use of different scaling techniques. Regarding situational covariation and similarity data, findings have indicated that the latter may be based on actual emotion co-occurrence (Schimmack \& Reisenzein, 1997). This implies that the two data types should correspond, which has been empirically demonstrated by Reisenzein and Schimmack (1999), as well as by Feldman Barrett and Fossum (2001). To be sure, the latter authors emphasize that similarity judgments are not exclusively based on episodic emotion information, but on semantic information as well.

The aim of our first study was to examine whether previous findings that suggest a high degree of correspondence between individual differences and situational covariation data on the one hand and similarity judgments on the other hand can be replicated and extended by examining whether the associations as implied by both covariation data types also mutually correspond. Additionally, we explored on what basis similarity judgments are made in order to gain some insight into possible associations between them and the other data types.

\section{STUDY 1}

Study 1 aimed to determine whether different kinds of data-individual differences covariation, situational covariation, and similarity judgments - imply similar associations 
between negative emotions. Regarding both types of covariation data, data from an earlier study by Kuppens, Van Mechelen, Smits, and De Boeck (2003) are used (see also Smits, De Boeck, Kuppens, \& Van Mechelen, 2002); the similarity data are new data collected for use in the present study.

\section{Method}

\section{Covariation data}

Participants. Participants were 175 12th-grade high school students from three different high schools, all located in the Dutch-speaking part of Belgium. Fourteen students did not adhere to the instructions, ${ }^{1}$ reducing the final sample size to 161 , which included 85 boys and 76 girls. The mean age was 17.4 years.

Materials and procedure. By means of a questionnaire, each participant was first asked to describe three recently experienced unpleasant situations that were all three characterized by either the absence or presence of one out of six prespecified features: appraisal of frustration, other accountability, arrogant entitlement, antagonistic action tendency, anger, or irritation. Twelve different types of questionnaire were used (presence/absence of one of the six prespecified features), and each participant was randomly assigned to one of them. Because this study focused on negative emotions, the instruction to describe 'unpleasant' situations was included to avoid descriptions of less relevant situations. The manipulation of the generation of the situations was primarily included to enable the construction of patterns of components (see study 2 below). In order to guarantee a certain degree of variation in the collected situations, in each questionnaire one of the three situations had to be sampled from the domain of work or school, one from the domain of interaction with close personal friends or family, and one from the domain of leisure time.

After having described all three situations, participants were asked successively for each of their three situation descriptions to imagine as vividly as possible what they had thought, how they had felt, and what they had wanted to do, and to indicate to which degree they had experienced a list of negative emotions in each of the situations. This list of negative emotions was taken from Diener et al. (1995) and was constructed to represent the domain of negative emotions. It consisted of 16 emotion labels, grouped into four categories: (i) fear (fear, worry, anxiety, and nervous), (ii) anger (anger, irritation, disgust, and rage), (iii) shame (shame, guilt, regret, and embarrassment), and (iv) sadness (sadness, loneliness, unhappiness, and depression). In order to obtain a comparable Dutch list, the 16 emotion labels were translated and back-translated twice: once by means of an EnglishDutch and Dutch-English dictionary, and once by two professional bilingual translators (one of whom was a native English speaker). The 16 Dutch emotion labels that converged through both the translation procedures were included in the final Dutch list, to be used in the questionnaires. In each questionnaire, participants had to indicate on a four-point scale (ranging from ' 0 ', not, to ' 3 ', very strongly) to which degree they had experienced each emotion from the list in each situation. Each participant filled out the questionnaire within the time limit of one class-hour (50 minutes). At the end, participants were debriefed about the purpose of the study.

\footnotetext{
${ }^{1}$ Non-adherence was defined as incompletely filled out questionnaires, situation descriptions that contained no reference to the instructions (e.g. hoax stories), or emotion ratings that showed deliberate bias (e.g. improbable patterns of ratings such as a clear zig-zag pattern).
} 


\section{Similarity ratings}

Participants. The participants who provided the similarity data were recruited on a voluntary basis. The group consisted of 30 psychology students, 25 female and 5 male. The mean age was 23.5 years.

Materials and procedure. The similarity ratings were collected for a list of 11 emotion terms that were included both in the list mentioned above and in the research of Frijda, Kuipers, and ter Schure (1989) (see study 2 below): fear, anxiety, nervous, anger, annoyance, disgust, rage, shame, guilt, regret, and sadness. Note that all four negative emotion categories from Diener et al. (1995), from which the emotion terms used in this study were taken, were represented.

The total of 55 possible emotion pairs was presented after one another using a computer, the order of presentation being randomized within and between emotion pairs. Subsequently, for each emotion pair, the participants were asked to indicate on a sevenpoint scale (ranging from ' 1 ', totally similar, to ' 7 ', totally different) to which degree the two emotions differed from one another. After the participants had finished their task, they were asked by means of a standard open-ended question on what basis they felt they had made their similarity judgments.

\section{Analyses and results}

After the omission of erroneous and invalid situation descriptions, a total of 443 valid situation descriptions was obtained from the 161 participants who provided the covariation data. From these data, several correlation indices between pairwise combinations of emotions were calculated. The correlations were calculated after specific preprocessing of the data. In particular, (i) global covariation information was obtained through calculating pairwise correlations between the emotions across all combinations of situations and persons $(N=437-443$, depending on missing data). The average observed correlation was $r=0.17 \quad(\mathrm{SD}=0.22)$. The data on which these correlations were calculated were not fully statistically independent; this was unproblematic, however, because the correlations in question were considered descriptive measures only. The obtained matrix of global intercorrelations was based on individual differences and situational variance, and was similar to the matrices typically used in previous emotion structure research. In the present study, however, we also sought to examine the issues at hand with respect to pure measures of individual differences emotion covariation and pure measures of situational emotion covariation. Therefore, matrices containing such information were also included. (ii) Individual differences covariation was obtained through averaging, for each participant separately, the emotion ratings over his or her three described situations. In this way, the variance stemming from situational differences was averaged out in order to minimize the confounding of individual differences and situational variance. Next, correlations were calculated for all pairwise emotion combinations across participants $(N=160-161$, depending on missing data). The average correlation was $r=0.24(\mathrm{SD}=0.23)$. (iii) Situational covariation data were obtained by using 12 different types of experimental questionnaires (based on presence/absence of one out of six prespecified situation features); moreover, each participant had to generate a situation from each of three domains (work/study, personal relations, and leisure); therefore, in total, 36 different types of situation could be distinguished. Raw emotion ratings were averaged across participants for each situation 
Table 1. Correlations between global covariation, individual differences covariation, situational covariation, and judged similarity between emotions (study 1)

\begin{tabular}{lcccc}
\hline & 1 & 2 & 3 & 4 \\
\hline 1. Global covariation & - & $0.98^{*}$ & $0.85^{*}$ & $0.85^{*}$ \\
2. Individual differences covariation & & - & $0.83^{*}$ & $0.83^{*}$ \\
3. Situational covariation & & & - & $0.72^{*}$ \\
4. Judged similarity & & & & - \\
\hline
\end{tabular}

$* p<0.0001, N=55$.

type so as to remove individual differences variance from the ratings. Next, correlations were calculated between emotion pairs across the 36 situation types. The average correlation was $r=0.23(\mathrm{SD}=0.31)^{2}$

With respect to the similarity ratings, interrater reliability was calculated across the 30 participants by means of Cronbach's alpha coefficient. The latter amounted to 0.95, implying a high inter-rater agreement. Next, the mean judged similarity was calculated for each emotion pair across all 30 participants. These mean values were subtracted from the maximum possible value (7) such that higher values reflect higher similarity. The average similarity between two emotions was $2.39(\mathrm{SD}=0.92)$.

Finally, correlations between global covariation, individual differences covariation, situational covariation, and average similarity judgments were calculated across the 55 emotion pairs for which similarity judgments were available. The results are shown in Table 1. The high correlations that are presented in this table imply that for any two association measures it holds that if two emotions are strongly associated according to the first measure then they will also be strongly associated according to the second, and vice versa. Given probable violations of statistical independence, caution is advised when interpreting the significance levels of the reported correlations.

Regarding the open-ended question pertaining to the participants' basis of the similarity judgment task, 26 out of the 30 participants provided one or more answers to it. These answers were divided into codable units (separate explanations), of which a total of 42 was obtained. Content analysis of these codable units yielded five categories of grounds for similarity judgments: (i) a specific relation between two emotions, such as cause-effect (one emotion causes the other to occur) or categorical inclusion (one emotion is part of the other emotion), (ii) co-occurrence of two emotions in the same situation (real-life, imagined, or in general), (iii) similarity in intensity, (iv) shared components, meaning, or action tendencies, and (v) subjective compatibility of the underlying experienced feelings (the subjective feelings underlying both emotions are reconcilable with one another). Table 2 shows the frequencies and percentages of occurrence for all categories.

The results showed that in $45 \%$ of the units reference was made to co-occurrence as a ground for the similarity judgments; moreover, this category was used by 19 of the 26 participants (73\%). Compatibility of underlying feelings is referred to in about one-quarter of the units, references to a specific relation or common underlying components occurred in $14 \%$ and $12 \%$ (respectively) of the units, whereas references to similar intensity were rather scarce.

\footnotetext{
${ }^{2}$ The complete association matrices containing global, individual differences and situational emotion covariation as well as the averaged emotion similarity matrix can be obtained on request from the first author.
} 
Table 2. Frequency and percentage of coding categories for the answers to the open-ended question 'On what did you base your similarity judgments?' (Study 1)

\begin{tabular}{lcc}
\hline Coding category & Frequency & Percentage (\%) \\
\hline Specific relation & 6 & 14 \\
Co-occurrence & 19 & 45 \\
Similarity in intensity & 2 & 5 \\
Common components & 5 & 12 \\
Compatible underlying feelings & 10 & 24 \\
Total & 42 & 100 \\
\hline
\end{tabular}

Several of the obtained categories can be combined into broader categories referring to either semantic information (common components, similar intensity) or to episodic information (co-occurrence, compatible feelings). According to this classification, only $17 \%$ of the answers referred to semantic grounds of similarity, whereas $69 \%$ of the answers referred to episodic explanations.

\section{Discussion}

The results clearly demonstrate that the associations between emotions implied by the various data types show strong correspondence. Part of this finding replicates findings from previous research. In particular, the correspondence between individual differences and situational covariation data on the one hand and similarity ratings on the other hand was also obtained in studies from Reisenzein and Schimmack (1999) and Feldman Barrett and Fossum (2001). The obtained correspondence values between emotion covariation and average emotion similarity were even higher than in previous studies in which similar methods were used (e.g. in the study by Reisenzein \& Schimmack a correlation of $r=0.53$ was obtained for negative emotions).

Moreover, our findings are also in line with participants' own introspective accounts of the basis for their similarity judgments. The majority of participants clearly referred to the possibility of two emotions co-occurring as a basis for their judged similarity. In more general terms, a vast majority of reported grounds of similarity judgments referred to episodic explanations. As such, this result supports the assertion made by Schimmack and Reisenzein (1997) that similarity judgments are chiefly based on an episodic memory of past emotional experiences. The answers to the open-ended question, however, also showed references to other, semantic grounds of similarity ratings, possibly ruling out an exclusive episodic account. For instance, participants also explicitly referred to common components as a basis for their similarity judgments. One may speculate that such components relate to dimensions or features that are hypothesized to determine semantic emotion knowledge; the latter would imply that similarity ratings are based on semantic knowledge as well (see also Feldman Barrett \& Fossum, 2001).

Beyond a replication of previous findings, it was shown that emotion associations implied by individual differences and situational covariation data show strong correspondence as well. This suggests that personality variables affect emotional experience in a similar way as situational variables do, which at first sight may seem rather surprising. A possible explanation for this may be that a common mechanism underlies both. Such a conjecture will be examined in the second part of this article. 


\section{PART 2: COMPONENTIAL BASIS OF ASSOCIATIONS BETWEEN EMOTIONS}

\section{Componential basis of emotion covariation}

The findings from study 1 may be considered to provide indirect evidence about processes that may lie at the base of emotional experience. Specifically, the strong correspondence between emotion associations as implied by individual differences and situational covariation data may reflect the fact that both types of association reflect the amount of component overlap between the emotions under study. Indeed, if one assumes that an emotion results from an additive combination of a number of components and makes some simplifying assumptions, then it can be shown that the correlation between two emotions, be it across persons or situations, is a direct function of the ratio of the number of common components to the total number of components of both emotions (the latter being the sum of common and distinctive components).

In particular, let $E_{1}$ and $E_{2}$ denote two emotions, and $A_{1}, A_{2}$, etc. denote emotion components, where $c$ is the number of common components, and $n$ and $m$ the numbers of distinct components of emotions $E_{1}$ and $E_{2}$, respectively (see the appendix for details). Assume further that an emotion equals to the additive combination of its components - that is,

$$
\begin{aligned}
& E_{1}=A_{1}+A_{2}+\cdots+A_{c}+A_{c+1}+\cdots+A_{c+n} \\
& E_{2}=A_{1}+A_{2}+\cdots+A_{c}+A_{c+n+1}+\cdots+A_{c+n+m}
\end{aligned}
$$

If all of the components are independent and distributed with identical variance, then it follows that

$$
\rho_{E_{1}, E_{2}}=\frac{c}{\sqrt{(c+n)(c+m)}}
$$

(see the appendix). Therefore, the correlation between the two emotions is a direct function of the ratio of the number of common components and the total number of components of each emotion. To be sure, this only holds strictly true if the above simplifying assumptions are made. It can be shown, however, that after a relaxation of these assumptions similar conclusions could be derived. Thus, when two emotions share most of their components, they will more likely covary then when they do not. Of course, if both individual differences and situational covariation reflect component overlap, then a strong correspondence between the two types of covariation immediately follows.

\section{Basis of similarity judgments}

Regarding the basis for similarity judgments, several conjectures can be advanced. A first account of similarity judgments is that they are based on semantic emotion information (see e.g. Feldman Barrett \& Fossum, 2001; Russell \& Fehr, 1994; Shaver et al., 1987). From this view, one may hypothesize that similarity judgments are directly related to component overlap, which can be viewed as essentially a comparison of defining features of the emotions. If emotion covariation is indeed based on component overlap, then this account predicts that the correspondence between emotion covariation and similarity judgments should disappear when controlling for component overlap. An alternative, episodic account states that similarity judgments are directly based on a person's intuitive impressions of emotion covariation based on episodic memory (Schimmack \& Reisenzein, 1997). In this case, the relation between similarity judgments and component overlap is expected to be mediated by emotion covariation, given our hypothesis that emotion covariation itself reflects component overlap. Finally, as a combination of both models, 
one may hypothesize that similarity judgments can be directly based on both semantic and covariation information. This possibility is suggested by the finding that the participants in study 1 mentioned both semantic and episodic information. Furthermore, this possibility is compatible with previous data (see e.g. Feldman Barrett \& Fossum, 2001; Reisenzein \& Schimmack, 1999).

An important question when considering the componential basis for emotion covariation and similarity judgments is which kind of components should be considered. In the present study, we will focus on appraisal and action tendency components, which are rooted in appraisal theories of emotion. The latter approach is widely regarded as a valid account of the elicitation and differentiation of emotions. Indeed, the high degree of convergence regarding which components are associated with which emotions reached in different studies by different researchers may rightfully be seen as a strong indicator of the validity of this approach. For instance, Scherer (1997) argued that the accumulated evidence provides substantial support for many of the theoretical claims of appraisal theorists (p. 115), and Frijda et al. (1989) pointed to 'the sizeable support for this [appraisal theory] approach, demonstrating strong relations between emotions and cognitive appraisal structures' (p. 212). In short, appraisal theories of emotion posit that different emotions are linked to (or elicited by) distinct patterns of cognitive appraisals (see e.g. Frijda, 1986, 1994; Ortony, Clore, \& Collins, 1988; Roseman, 1984; Scherer, 1993; Smith \& Ellsworth, 1985; Smith \& Lazarus, 1993). Furthermore, within this approach it has been argued that, in addition to appraisals, action tendencies should also be considered as distinctive emotion components (see e.g. Fischer, 1991; Frijda, 1986; Frijda et al., 1989; Lazarus, 1991; Oatley \& Jenkins, 1996; Skiffington, Fernandez, \& McFarland, 1998).

Partial empirical corroboration of the existence of a relation between component overlap, judged similarity, and (individual differences) emotion covariation was provided by Frijda (1987). He constructed several separate matrices that each reflected a particular type of association between a set of emotions - overlap in appraisal patterns, overlap in patterns of action tendencies, emotion similarity (based on a word sorting task), and correlations across persons between mood-adjective checklist items. On each matrix, a separate multidimensional scaling (MDS) analysis was performed. For each type of emotion association, this yielded dimension coordinates for each emotion term. Subsequently, for each combination of two types of emotion association, a canonical correlation analysis was performed to determine the degree of correspondence between the two MDS solutions. The results demonstrated that the structures of the appraisal and action tendency overlap matrices strongly resembled those of the emotion similarity and correlation matrices (Frijda, 1987). A limitation of Frijda's study, however, was that he examined the degree of correspondence between structural representations (based on various types of emotion association). A direct way to examine our hypothesis would involve comparing the raw association data without subjecting them to data analyses prior to the comparison. Also, Frijda did not evaluate any explicit hypotheses regarding the basis of similarity judgments.

In the following study, it will be directly examined whether the associations between emotions, as implied by global, individual differences and situational covariation data and by similarity ratings, are related to the degree of overlap between patterns of underlying components. Furthermore, alternative conjectures regarding the basis of similarity judgments will be evaluated. 
In this study, we made use of two different sets of components to establish measures of component overlap between the emotions. A first set of components was taken from the study by Kuppens et al. (2003) on which the emotion covariation data were based. On the basis of previous research, Kuppens et al. (2003) selected appraisal and action tendency aspects relevant for negative emotions - anger in particular. These aspects included the appraisals of frustration, other accountability and arrogant entitlement, and an antagonistic action tendency. By means of these aspects, the authors constructed patterns of components for the same list of negative emotions as used in study 1 . The patterns provided clear distinctions between the emotion categories of fear, anger, sadness, and shame (Kuppens et al., 2003) and were used in the present study.

The use of these patterns to establish component overlap implies two caveats. First, the correlations that provided the global, individual difference, and situational covariation measures from study 1 were calculated in part on the basis of the same data (emotion ratings) as the component patterns (vectors of associations between emotion ratings and component variables). Thus, correspondence between the former and the latter may to some extent be due to overlap in the data. Second, the appraisal and action tendency aspects from Kuppens et al. (2003) were primarily selected on the basis of their relevance for anger. As such, they may not always be relevant for the other included negative emotions, for which other appraisal and action tendency aspects might be more relevant. The latter may obscure the true extent of overlap between the emotions' underlying components.

Following from these considerations, we included a second set of components, which was adopted from a study by Frijda et al. (1989). In the latter study, appraisal patterns as well as action tendency patterns were constructed for a set of 32 emotion terms, including 19 negative, 3 neutral, and 11 positive terms. The patterns consisted of 13 appraisal and 13 action tendency aspects and showed distinct relations to various emotion categories and single emotion terms. By including this set of components, data overlap was strictly avoided, and components relevant for each emotion under study were included.

\section{STUDY 2}

The emotion covariation and similarity data that were used in this study were taken from study 1 . The following method section will discuss how patterns of components were constructed in the research by Kuppens et al. (2003) and Frijda et al. (1989), respectively.

\section{Method}

Component patterns from Kuppens et al. (2003)

In the research by Kuppens et al. (2003), 161 participants provided emotion ratings for three recently experienced unpleasant situations. The instructions asked participants to recall specific types of situations - namely, situations in which one of the following aspects was either present or absent: frustration, other accountability, arrogant entitlement, antagonistic action tendency, anger, or irritation. Thus, in total there were 12 different types of experimental questionnaires [presence/absence $\times(4$ components +2 emotions $)$ ], and each participant was randomly assigned one of them. In order to construct patterns of components associated with each of the emotions, association indices $\varphi$ (Fleiss, 1981) were calculated for each combination of the 11 emotions and the 4 components, the indices representing the degree of association between the components and the emotions. 
To this end, the data were first dichotomized - ' 0 ' and ' 1 ' were scored as ' 0 ', and ' 2 ' and ' 3 ' were scored as ' 1 ' - allowing for the construction of two by two contingency tables (presence/absence of component, based on the questionnaire instructions, and presence/ absence of emotion), on which the association indices were calculated. The $\varphi$ index ranges from -1 (perfect negative association) to +1 (perfect positive association).

\section{Component patterns from Frijda et al. (1989)}

In the study by Frijda et al. (1989), participants had to rate recalled emotion episodes on a seven-point scale with respect to 13 appraisal and 13 action tendency aspects (the scales were bipolar in the case of appraisal aspects and unipolar in the case of action tendency aspects). The authors then derived a code for the degree of association between each aspect and each emotion term: ' + ' (respectively ' ++ ') if the average appraisal or action tendency rating was 1 (respectively 2 or more) points above the scale midpoint, ' - ' (respectively ' - - ') if the average appraisal rating was 1 (respectively 2 or more) points below the scale midpoint, and 0 otherwise. In the present study, such indices were replaced by the values of $1,2,-1,-2$, and 0 , respectively, denoting the strength of the emotioncomponent associations. The authors were contacted and asked for the precise Dutch labels of the emotion terms used in Frijda et al. (1989). Given that the latter study and our own study were both conducted in Dutch, an exact matching of emotion terms was possible. Eleven negative emotion labels were identified that were used in study 1 , in study 2, and in the research by Frijda et al. (1989).

Component patterns from both studies were constructed in different ways. For the data from Kuppens et al. (2003), no component ratings were collected, and emotion-component associations were calculated on the basis of contingency tables resulting from emotion ratings and questionnaire instructions. For the data from Frijda et al. (1989), no emotion ratings were available, and mean component scores were calculated for each emotion (recoded as $-2,-1,0,1$ and 2). The types of data collected in both studies do not allow for an identical construction of component patterns. Both methods, however, result in describing the strength of a particular component-emotion relation. Therefore, we do not believe that this methodological difference had an impact on the results of the present study.

\section{Results}

Two measures of component overlap were calculated, one based on the component patterns from Kuppens et al. (2003) and one based on those from Frijda et al. (1989). In each case, the Euclidean distance between the component patterns of each emotion pair was calculated as a measure of component overlap. A direct measure of overlap for each set of components was then obtained by subtracting the Euclidean distances from $0 .^{3}$ The

\footnotetext{
${ }^{3}$ As an example, we will illustrate this calculation for the emotions of anger and guilt with component patterns from Kuppens et al. (2003). In the latter study, the $\varphi$-values reflecting the associations between anger and guilt on the one hand and the components of frustration, other accountability, arrogant entitlement, and antagonism on the other hand were $0.39,0.23,0.23$, and 0.01 , and $-0.03,-0.23,-0.21$, and 0.13 , respectively. The Euclidean distance between the component patterns of the emotions is calculated as follows:

$$
d_{\text {anger,guilt }}=\sqrt{[0.39-(-0.03)]^{2}+[0.23-(-0.23)]^{2}+[0.23-(-0.21)]^{2}+[0.01-0.13]^{2}}=0.77
$$

To transform the resulting distance value $d$ into a measure of overlap $o$, it is subtracted from zero $\left(o_{\text {anger,guilt }}=-0.77\right)$.
} 
Table 3. Correlations between on the one hand global covariation, individual differences covariation, situational covariation, and judged similarity between emotions, and on the other hand measures of component overlap based on component patterns taken from Kuppens et al. (2003), and Frijda et al. (1989), respectively (Study 2)

\begin{tabular}{lcc}
\hline & $\begin{array}{c}\text { Component overlap } \\
\text { Kuppens et al. }\end{array}$ & $\begin{array}{c}\text { Component overlap } \\
\text { Frijda et al. }\end{array}$ \\
\hline Global covariation & $0.74 * *$ & $0.44 * *$ \\
Individual differences covariation & $0.77 * *$ & $0.37 *$ \\
Situational covariation & $0.73 * *$ & $0.36 *$ \\
Judged similarity & $0.64 * *$ & $0.52 * *$ \\
\hline
\end{tabular}

$* p<0.01 ; * * p<0.001, N=55$.

correlation between the two measures of overlap across emotion pairs was $r=0.33$ $(p<0.05)$.

The global covariation, individual differences covariation, situational covariation, and similarity measures from study 1 , on the one hand, and the overlap measures, on the other hand, were correlated across all 55 emotion pairs (Table 3). All correlations were high and positive, meaning that the associations between emotions obtained in study 1 were strongly associated with the degree of overlap between their underlying components.

To address the question of the basis of similarity judgments, multiple regression analyses were performed. Each analysis included global covariation between emotions (representing general co-occurrence of emotions or episodic information) and one of the two measures of component overlap (representing semantic information) as predictor variables, and the averaged similarity judgments as a criterion variable (Table 4). On the one hand, the results clearly show that similarity judgments are directly and strongly related to emotion covariation-comparing the standardized regression weights to the correlations reported in Table 1, the correspondence between emotion covariation and similarity does not decrease strongly when controlling for component overlap. On the other hand, the high correlations between similarity judgments and component overlap reported in Table 1 do not remain when controlling for emotion covariation: For component overlap based on the patterns from Kuppens et al. (2003), the correspondence disappears; for component overlap based on patterns from Frijda et al. (1989), the

Table 4. Summary of multiple regression analyses predicting averaged similarity judgments of emotions $(N=55)$, using global covariation and component overlap based on Kuppens et al. (2003) (analysis 1), and global covariation and component overlap based on Frijda et al. (1989) (analysis 2) as predictor variables (Study 2)

\begin{tabular}{lcccc}
\hline Predictor variables & $B$ & SE $B$ & $\beta$ & $p$ \\
\hline Analysis 1 & & & & \\
$\quad$ Global covariation & 3.53 & 0.45 & 0.85 & 0.00 \\
$\quad$ Component overlap Kuppens et al. & 0.01 & 0.45 & 0.00 & 0.98 \\
Analysis 2 & & & & \\
$\quad$ Global covariation & 3.21 & 0.32 & 0.77 & 0.00 \\
$\quad$ Component overlap Frijda et al. & 0.24 & 0.10 & 0.18 & 0.02 \\
\hline
\end{tabular}

$R^{2}=0.72$ for analysis $1 ; R^{2}=0.75$ for analysis 2 . 
correspondence strongly weakens, although it remains statistically significant. As such, the results disconfirm an exclusive semantic model of similarity judgments.

\section{Discussion}

This study demonstrates that various types of association between negative emotions can, at least in part, be accounted for by means of their underlying component patterns, consisting of appraisals and action tendency aspects. Emotions that show more overlap with respect to their underlying components are more likely to covary with one another (on an individual differences level as well as on a situational level) and are judged to be more similar to one another. With respect to emotion covariation, our finding is in line with claims of other authors, such as Diener et al. (1995), who suggested that the basis for long-term affect structure may be found in evaluative or appraisal processes. With respect to similarity judgments, the results from the regression analyses suggest that similarity judgments of emotions primarily reflect emotion covariation instead of component overlap. Similarity judgments, however, cannot be entirely reduced to such covariation-when one takes into account a broad variety of components that are relevant for all emotions studied, component overlap directly contributes to the judged similarity of emotions, albeit only to a small degree. As such, the findings strikingly correspond to intuitive accounts of participants regarding their emotion similarity judgments from study 1 .

As an aside, we would like to note that the obtained correspondence between emotion covariation and component overlap based on patterns from Kuppens et al. (2003) cannot be entirely due to data overlap, given the substantial correlations obtained with the other set of components from Frijda et al. (1989). The fact that the correlations based on the latter data are somewhat lower than those based on the former might be due in part to differences between participant groups and to differences in time lag between the collection of the data from which the associations between the emotions were derived and the collection of the data on the component patterns. In the former case both types of data were collected with Belgian students and the time lag was less than one year, whereas in the latter case the two types of data were collected from Belgian and Dutch students, respectively, and the time lag was more then 10 years.

\section{GENERAL DISCUSSION}

In the presented research, we found empirical corroboration for a high degree of correspondence between emotion associations as implied by individual differences covariation, situational covariation, and similarity data. These findings suggest that a common ground may be assumed underlying emotion associations as implied by different data types. In this respect, it was theoretically demonstrated that if an emotion can be assumed to be an additive combination of a number of basic components, then associations between emotions as implied by the different types of data should reflect component overlap and, hence, should show high mutual correspondence. Furthermore, the findings from study 2 provide direct evidence that all the types of emotion associations mentioned above are related to overlap in appraisals and action tendencies. The latter finding replicates and extends (with respect to situational covariation data) findings from Frijda (1987); it is also in line with the assertion made by Russell (1980) that the same processes that lie at the base of one's own actual emotional experiences may also contribute to the organization of one's knowledge about emotions. 
Taken together, the findings thus suggest that the component process is involved, in a direct or indirect way, in emotion elicitation in specific situations, in specific emotion traits, and in similarity judgments of emotions. As an aside, we would like to note that analogous findings have been reported in the field of personality psychology, with respect to covariation and similarity of traits and act overlap (see e.g. Borkenau, 1990, 1992).

The relation between individual differences covariation of emotions and component overlap further suggests the existence of stable individual differences in the occurrence of the components as a basis for individual differences in emotional experience. Indeed, if an emotion results from a combination of components and if there are individual differences in the propensity to experience various emotions (such as, for instance, trait anger or trait anxiety, Spielberger \& Sydeman, 1994; or, in general, negative affectivity, Watson, 2000), then the results indicate that the latter could be based on individual differences in experiencing the components associated with the particular emotion or affect. The latter type of individual differences refers to tendencies to experience certain appraisals. Such appraisal tendencies, including, for instance, hostile attribution bias, have already been described elsewhere by several authors (Dodge, 1993; Griner \& Smith, 2000; Matthews, Derryberry, \& Siegle, 2000), and have been linked to several sources of individual differences, including biologically based ones (see van Reekum \& Scherer, 1997); they may provide a promising lead for future research addressing individual differences in emotional experience.

In a similar way, the found relation between situational covariation and component overlap suggests that systematic differences between situations regarding the ease with which they elicit emotion components may underlie situational differences in the occurrence of emotions. Note that the existence of such differences between situations is fully in line with the notion of active psychological features of situations used in contextualized personality psychology (Mischel \& Shoda, 1995; Shoda, Mischel, \& Wright, 1993; Vansteelandt \& Van Mechelen, 1998); this notion implies that situations differ from one another in terms of characteristics that have a systematic impact on the behaviors or emotions that are elicited by them.

Finally, from our findings on similarity judgments, we may conclude that such judgments may primarily be based on the co-occurrence of emotions, in line with research by Schimmack and Reisenzein (1997). Although a strong correspondence between similarity judgments and component overlap was found as well, this relation seemed to be mediated by emotion covariation to a considerable extent. Still, the results from the regression analysis using a broad array of components in determining component overlap in study 2 , as well as the introspective accounts from the similarity judges themselves in study 1 , did not completely rule out the possibility that similarity judgments can in part be directly based on a comparison of defining features of the emotions as well (see also Feldman Barrett \& Fossum, 2001).

In sum, the present study provided evidence demonstrating that data reflecting individual differences covariation of emotions, data reflecting situational covariation of emotions, and data reflecting emotion similarity judgments imply similar associations between emotions. Moreover, it was theoretically argued and empirically confirmed that overlap between emotions' appraisals and action tendencies may lie at the base of the emotion associations implied by the different covariation data types, thus explaining the high degree of correspondence between the data types. Similarity judgments, in turn, seemed to be primarily based on emotion covariation, and are only to a smaller degree directly related to component overlap. 


\section{ACKNOWLEDGEMENTS}

The authors wish to thank Nico Frijda for supplying the Dutch translations of emotion terms from Frijda et al. (1989), and Scott Acton, Eva Ceulemans, Johnny Fontaine, and Kristof Vansteelandt for their valued comments on a previous version of this paper.

\section{APPENDIX}

The derivation can be obtained as follows:

$$
\begin{aligned}
\rho_{E_{1}, E_{2}} & =\frac{\sigma_{E_{1}, E_{2}}}{\sigma_{E_{1}} \sigma_{E_{2}}} \\
& =\frac{\sigma_{A_{1}+\cdots+A_{c}+A_{c+1}+\cdots+A_{c+n}, A_{1}+\cdots+A_{c}+A_{c+n+1}+\cdots+A_{c+n+m}}}{\sqrt{\sigma_{A_{1}+\cdots+A_{c}+A_{c+1}+\cdots+A_{c+n}}^{2}} \sqrt{\sigma_{A_{1}+\cdots+A_{c}+A_{c+n+1}+\cdots+A_{c+n+m}}^{2}}}
\end{aligned}
$$

Assuming that the components are independent from one another (i.e., if $\sigma_{A_{i}, A_{j}}=0$ ), it follows that (with $\sigma_{A_{i}+A_{j}}^{2}=\sigma_{A_{i}}^{2}+\sigma_{A_{j}}^{2}+2 \sigma_{A_{i}, A_{j}}$ )

$$
\rho_{E_{1}, E_{2}}=\frac{\sum_{i=1}^{c} \sigma_{A_{i}, A_{i}}}{\sqrt{\sigma_{A_{1}}^{2}+\cdots+\sigma_{A_{c+n}}^{2}} \sqrt{\sigma_{A_{1}}^{2}+\cdots+\sigma_{A_{c}}^{2}+\sigma_{A_{c+n+1}}^{2}+\cdots+\sigma_{A_{c+n+m}}^{2}}}
$$

If we further assume that the components share identical variances, it follows that

$$
\begin{aligned}
\rho_{E_{1}, E_{2}} & =\frac{c \sigma_{A}^{2}}{\sqrt{(c+n)(c+m)} \sigma_{A}^{2}} \\
& =\frac{c}{\sqrt{(c+n)(c+m)}}
\end{aligned}
$$

\section{REFERENCES}

Borkenau, P. (1990). Systematic distortion and systematic overlap in personality ratings. In G. L. Van Heck, S. E. Hampson, J. Reykowski, \& J. Zakrzewski (Eds.), Personality psychology in Europe: Foundations, models and inquiries (Vol. 3, pp. 3-29). Lisse, The Netherlands: Swets \& Zeitlinger.

Borkenau, P. (1992). Implicit personality theory and the five-factor model. Journal of Personality, 60, 295-327.

Coren, S., \& Russell, J. A. (1992). The relative dominance of different facial expressions of emotion under conditions of perceptual ambiguity. Cognition and Emotion, 6, 339-356.

Diener, E. (1999). Introduction to the special section on the structure of emotion. Journal of Personality and Social Psychology, 76, 803-804.

Diener, E., Smith, H., \& Fujita, F. (1995). The personality structure of affect. Journal of Personality and Social Psychology, 69, 130-141.

Dodge, K. A. (1993). Social-cognitive mechanisms in the development of conduct disorder and depression. Annual Review of Psychology, 44, 559-584.

Feldman Barrett, L., \& Fossum, T. (2001). Mental representations of affect knowledge. Cognition and Emotion, 15, 333-363.

Fischer, A. H. (1991). Emotion scripts: A study of the social and cognitive facets of emotions. Leiden: DSWO Press.

Fleiss, J. L. (1981). Statistical methods for rates and proportions (2nd ed.). New York: Wiley.

Frijda, N. H. (1986). The emotions. Cambridge: Cambridge University Press. 
Frijda, N. H. (1987). Emotion, cognitive structure, and action tendency. Cognition and Emotion, 1, 115-143.

Frijda, N. H. (1994). Emotions are functional, most of the time. In P. Ekman, \& R. J. Davidson (Eds.), The nature of emotion: Fundamental questions (pp. 112-122). New York: Oxford University Press.

Frijda, N. H., Kuipers, P., \& ter Schure, E. (1989). Relations among emotion, appraisal, and emotional action readiness. Journal of Personality and Social Psychology, 57, 212-228.

Griner, L. A., \& Smith, C. A. (2000). Contributions of motivational orientation to appraisal and emotion. Personality and Social Psychology Bulletin, 26, 727-740.

Kuppens, P., Van Mechelen, I., Smits, D. J. M., \& De Boeck, P. (2003). The appraisal basis of anger: Specificity, necessity and sufficiency of components. Emotion, 3, 254-269.

Lazarus, R. S. (1991). Cognition and motivation in emotion. American Psychologist, 46, 352-367.

Lazarus, R. S. (1994). Individual differences in emotion. In P. Ekman, \& R. J. Davidson (Eds.), The nature of emotion: Fundamental questions (pp. 332-336). New York: Oxford University Press.

Matthews, G., Derryberry, D., \& Siegle, G. J. (2000). Personality and emotion: Cognitive science perspectives. In S. E. Hampton (Ed.), Advances in personality psychology (Vol. 1, pp. 199-237). Hove, UK: Psychology Press.

Mischel, W., \& Shoda, Y. (1995). A cognitive-affective system theory of personality: Reconceptualizing situations, dispositions, dynamics, and invariance in personality structure. Psychological Review, 102, 246-268.

Morgan, R. L., \& Heise, D. (1988). Structure of emotions. Social Psychology Quarterly, 51, $19-31$.

Oatley, K., \& Jenkins, J. M. (1996). Understanding emotions. Oxford: Blackwell.

Ortony, A., Clore, G., \& Collins, A. (1988). The cognitive structure of emotions. New York: Cambridge University Press.

Reisenzein, R., \& Schimmack, U. (1999). Similarity judgments and covariations of affects: Findings and implications for affect structure research. Personality and Social Psychology Bulletin, 25, 539-555.

Roseman, I. J. (1984). Cognitive determinants of emotion: A structural theory. In P. Shaver (Ed.), Review of personality and social psychology: Emotions, relationships, and health (Vol. 5, pp. 11-36). Beverly Hills, CA: Sage.

Russell, J. A. (1980). A circumplex model of affect. Journal of Personality and Social Psychology, 39, 1151-1178.

Russell, J. A., \& Fehr, B. (1994). Fuzzy concepts in a fuzzy hierarchy: Varieties of anger. Journal of Personality and Social Psychology, 67, 186-205.

Scherer, K. R. (1993). Studying the emotion-antecedent appraisal process: An expert system approach. Cognition and Emotion, 7, 325-355.

Scherer, K. R. (1997). Profiles of emotion-antecedent appraisal: Testing theoretical predictions across cultures. Cognition and Emotion, 11, 113-150.

Schimmack, U., \& Reisenzein, R. (1997). Cognitive processes involved in similarity judgments of emotions. Journal of Personality and Social Psychology, 73, 645-661.

Shaver, P., Schwartz, J., Kirson, D., \& O’Connor, C. (1987). Emotion knowledge: Further exploration of a prototype approach. Journal of Personality and Social Psychology, 52, 1061-1086.

Shoda, Y., Mischel, W., \& Wright, J. C. (1993). The role of situational demands and cognitive competencies in behavior organization and personality coherence. Journal of Personality and Social Psychology, 65, 1023-1035.

Skiffington, S., Fernandez, E., \& McFarland, K. (1998). Towards a validation of multiple features in the assessment of emotions. European Journal of Psychological Assessment, 14, 202-210.

Smith, C. A., \& Ellsworth, P. C. (1985). Patterns of cognitive appraisal in emotion. Journal of Personality and Social Psychology, 48, 813-838.

Smith, C. A., \& Lazarus, R. S. (1993). Appraisal components, core relational themes, and the emotions. Cognition and Emotion, 7, 233-269.

Smith, L. L. (2002). Roles, identities, and emotions: Parallel processing and the production of mixed emotions. In Y. Kashima, M. Foddy, \& M. J. Platow (Eds.), Self and identity: Personal, social and symbolic (pp. 125-143). Mahwah, NJ: Erlbaum.

Smits, D. J. M., De Boeck, P., Kuppens, P., \& Van Mechelen, I. (2002). The structure of negative emotion scales: Generalization over contexts and comprehensiveness. European Journal of Personality, 16, 127-141. 
Spielberger, C. D., \& Sydeman, S. J. (1994). State-trait anxiety inventory and state-trait anger expression inventory. In M. E. Maruish (Ed.), The use of psychological tests for treatment planning and outcome assessment (pp. 393-321). Hillsdale, NJ: LEA.

van Reekum, C. M., \& Scherer, K. R. (1997). Levels of processing in emotion-antecedent appraisal. In G. Matthews (Ed.), Cognitive science perspectives on personality and emotion (pp. 259-300). Amsterdam: Elsevier.

Vansteelandt, K., \& Van Mechelen, I. (1998). Individual differences in situation-behavior profiles: A triple typology model. Journal of Personality and Social Psychology, 75, 751-765.

Watson, D. (2000). Mood and temperament. New York: Guilford.

Watson, D., \& Tellegen, A. (1985). Towards a consensual structure of mood. Psychological Bulletin, 98, 219-235.

Williams, P., \& Aaker, J. L. (2002). Can mixed emotions peacefully coexist? Journal of Consumer Research, 28, 636-649.

Zelenski, J. M., \& Larsen, R. J. (2000). The distribution of basic emotions in everyday life: A state and trait perspective from experience sampling data. Journal of Research in Personality, 34, 178-197.

Zevon, M. A., \& Tellegen, A. (1982). The structure of mood change: An idiographic/ nomotheticanalysis. Journal of Personality and Social Psychology, 43, 111-122.

Zurbriggen, E. L., \& Sturman, T. S. (2002). Linking motives to emotions: A test of McClelland's hypotheses. Personality and Social Psychology Bulletin, 28, 521-535. 